\title{
Utilidad de la angioresonancia en la planificación preoperatoria de reconstrucción mamaria mediante colgajos libres abdominales DIEP, SIEA y TRAM*
}

\author{
Drs. DIEGO POLANCO M. ${ }^{1}$, JOSÉ FERNÁNDEZ-CAÑAMAQUE R. ${ }^{1}$, \\ ISABEL ORTEGA C. ${ }^{1}$, ERNESTO CASTILLO G. ${ }^{2}$ \\ 1 Servicio de Cirugía Plástica. \\ 2 Servicio de Radiología. \\ Hospital Universitario de Getafe. \\ Madrid, España.
}

\begin{abstract}
Usefulness of magnetic resonance angiography in the preoperative study for breast reconstruction using epigastric flaps

Background: Mammary reconstruction after surgery for breast cancer can be performed with free abdominal flaps such as DIEP (deep inferior epigastric perforator), TRAM (transverse rectus abdominis musculocutaneus) y SIEA (superficial inferior epigastric artery). Aim: To report our experience with the use of magnetic resonance angiography to plan mammary reconstruction. Material and Methods: The superficial epigastric vascular axis and the connections between the superficial and deep axis were explored with magnetic resonance angiography in 25 patients aged 32 to 63 years. Dominant perforating arteries (those with the greater caliber and predominant distribution through adipose tissue), were localized. Their intramuscular trajectory and that of the deep epigastric artery were also studied. The findings of magnetic resonance were compared with those of intraoperative Doppler ultrasound examination dissection during operation. Results: There was a $100 \%$ concordance between magnetic resonance findings and those of intraoperative Doppler. However in two patients a perforating artery with an adequate caliber was missed with magnetic resonance. Conclusions: Magnetic resonance angiography is useful in the planning of mammary reconstruction with epigastric flaps.
\end{abstract}

Key words: MR-Angiography, free flaps, breast reconstruction.

\section{Resumen}

Objetivos: Dar a conocer nuestra experiencia en la toma de decisiones para la reconstrucción mamaria con colgajos libres de tejido abdominal mediante angioresonancia. Materiales y Métodos: Nuestra muestra corresponde a 25 pacientes intervenidos entre los años 2007-2009. Mediante angioresonancia se exploró el eje vascular epigástrico superficial, así como las conexiones entre el sistema profundo y superficial, localizando las perforantes dominantes (las de mayor calibre y distribución predominante por la grasa), su trayecto

*Recibido el 22 de Abril de 2010 y aceptado para publicación el 17 de Mayo de 2010.

Correspondencia: Dr. Diego Polanco M.

Carretera Toledo 12500, CP: 28905. Getafe, Madrid. España.

E-mail:dpolanco@puc.cl 
intramuscular y estudiando el trayecto de la arteria epigástrica profunda. Resultados: Los hallazgos en la angioresonancia fueron utilizados en el marcaje preoperatorio y contrastados con los hallazgos evidenciados por la ultrasonografía y durante la disección intraoperatoria de los colgajos, siendo la información obtenida congruente en un $100 \%$ de los casos, lo que facilita la decisión del colgajo a disecar, acortando los tiempo operatorios y dando mayor seguridad al cirujano. Conclusiones: La angioresonancia es un método útil en el estudio preoperatorio otorgando el beneficio adicional de no irradiar a los pacientes.

Palabras clave: Angiografía, colgajos libres, reconstrucción mamaria.

\section{Introducción}

El cáncer de mama es una enfermedad con un alta prevalencia actualmente. Los esfuerzos sanitarios van destinados al diagnóstico precoz y al tratamiento oportuno, esto finalmente conlleva una diversidad de planteamientos terapéuticos como son la cirugía conservadora, mastectomía, linfadenectomía, quimio y radioterapia ${ }^{1}$.

Es por esto que la reconstrucción mamaria es un procedimiento cada vez más habitual para pacientes con secuelas de mastectomía o cirugía conservadora por cáncer de mama. Dentro de las alternativas terapéuticas ${ }^{2}$ se encuentran los colgajos pediculados miocutáneos (latisimus dorsi, TRAM), de perforantes de vasos intercostales o de vasos toracodorsales, uso de expansores y prótesis y colgajos libre. Sin embargo, el uso de colgajos libres de tejido abdominal posee ventajas dados la similitud de textura, color, ubicación de la cicatriz y el beneficio estético lógico que otorga a las pacientes ${ }^{1}$.

Dentro de los colgajos libres abdominales destacan el DIEP (deep inferior epigastric perforator), TRAM (transverse rectus abdominis musculocutaneus) y SIEA (superficial inferior epigastric artery). El colgajo DIEP descrito inicialmente por Koshima y Soeda el año $1989^{3}$, requiere una disección intramuscular, en ciertas oportunidades tediosa y técnicamente compleja, del vaso perforante hasta la arteria epigástrica profunda, sin sacrificar músculo recto abdominal ${ }^{4}$. El colgajo libre TRAM (MS2), lleva consigo una pequeña pastilla de músculo recto abdominal, lo que disminuye las secuelas funcionales comparado con el colgajo TRAM pediculado ${ }^{5}$. El colgajo SIEA, aunque descrito el año 1975 por Taylor y Daniel ${ }^{6}$, recién fue utilizado en reconstrucción mamaria por Grotting el año 19947, destaca por la ausencia de morbilidad en estructuras musculares al transcurrir en forma superficial a estos hasta la arteria femoral ${ }^{8}$. El colgajo DIEP posee ventajas con respecto al TRAM libre en cuanto a la morbilidad de la zona donante, por ejemplo, asimetrías abdominales, hernias, disminución de la fuerza para la flexión y rotación de tronco ${ }^{9}$.

El estudio preoperatorio básico es la ultrasonografia doppler ${ }^{10,11}$, sin embargo, esta posee ciertos inconvenientes como son el alto número de falsos positivos y considerables cifras de falsos negativos. Es por esto que actualmente el uso de tomografía axial ${ }^{1,12}$ y angioresonancia ${ }^{13}$, resultan métodos extraordinariamente útiles en la planificación preoperatoria de colgajos libres de tejido abdominal, teniendo un impacto en el tiempo de disección y en la selección del colgajo y su ubicación ${ }^{1,12}$. Teniendo en cuenta la exposición a radiación ionizante de la tomografía axial en el contexto de pacientes con cáncer, creemos que la angioresonancia es un método que también debe considerarse ${ }^{13}$. Presentamos a continuación nuestra experiencia con el uso de angioresonancia en el planeamiento preoperatorio en el Hospital Universitario de Getafe de Madrid.

\section{Material y Método}

Se presentan 25 casos de pacientes de sexo femenino intervenidos durante los años 2007 a 2009 mediante colgajos libres abdominales para reconstrucción mamaria. En 8 pacientes la reconstrucción fue inmediata, mientras que en 16 fue diferida (Tabla 1). En la planificación preoperatorio se ha utilizado la angioresonancia.

La interpretación de las imágenes y su procesamiento fueron realizadas por radiólogo experto en angioresonancia. Se realizan reconstrucciones con

Tabla 1. Características de los pacientes y procedimiento quirúrgico realizado

\begin{tabular}{lc}
\hline Número de pacientes & 25 \\
Edad promedio & 45 años (32-63) \\
Reconstrucción inmediata & 9 \\
Reconstrucción diferida & 16 \\
DIEP & 9 \\
TRAM MS2 & 15 \\
SIEA & 1 \\
\hline
\end{tabular}

DIEP: deep inferior epigastric perforator. TRAM: transverse rectus abdominis musculocutaneus. SIEA: superficial inferior epigastric artery. 


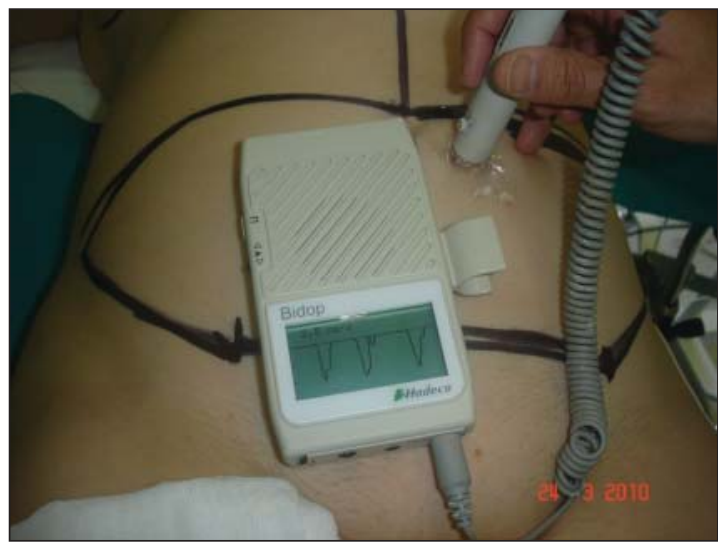

Figura 1. Diseño del colgajo y ubicación de vaso perforante mediante Doppler de acuerdo a la angioresonancia.

algoritmo MIP (Maximus intensity proyection) y cortes coronales y sagitales de la pared abdominal y su vascularización. Al mismo tiempo se identifican los vasos perforantes y sus coordenadas en relación al ombligo y la línea media. En conjunto con el cirujano plástico se estudia el diámetro de la perforante, su trayecto intramuscular y su ubicación respecto al ombligo, dando prioridad a las dos primeras variables descritas. Se explora el eje vascular epigástrico superficial, así como las conexiones entre los sistemas profundo y superficial, se localiza la perforante dominante (la de mayor calibre y distribución predominante por la grasa), si existe, y se estudia el trayecto de la arteria epigástrica profunda.

Los hallazgos en la angioresonancia fueron utilizados en el marcaje preoperatorio, en la toma de decisiones respecto al colgajo a disecar y posteriormente fueron contrastados con los hallazgos evidenciados por la ultrasonografía (Figura 1) y durante la disección intraoperatoria de los colgajos (Figura 2).

\section{Resultados}

Si se evidencia un eje vascular dominante superficial, sobre todo si hay arteria epigástrica inferior superficial de un calibre superior a $1,5 \mathrm{~mm}$, la decisión preoperatoria se orienta a SIEA (Figura 3), puesto que es el colgajo de elección y que menor morbilidad causa a la pared abdominal. Si la arteria tiene un calibre menor, pero con dominancia del sistema venoso superficial, decidimos realizar habitualmente un TRAM MS2 o un colgajo DIEP, dejando al menos una vena superficial disecada, clipada y preparada para una posible segunda anastomosis venosa, garantizando un mejor retorno venoso al colgajo (siendo esta segunda opción algo más com-

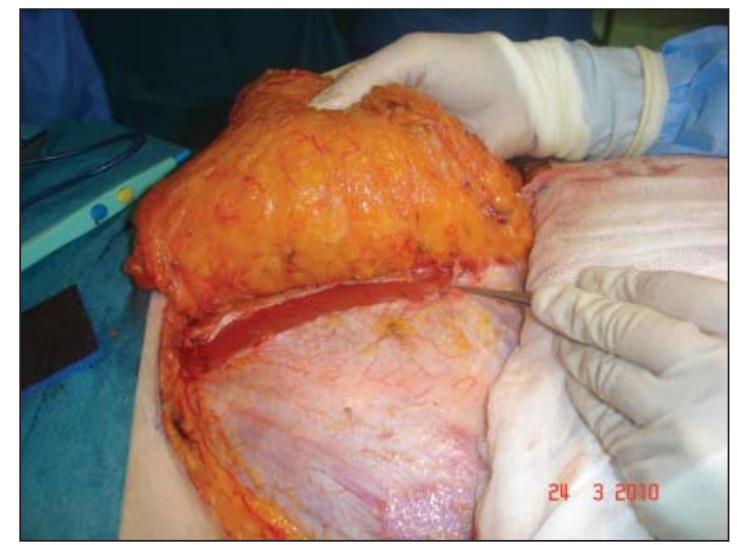

Figura 2. Disección de vaso perforante previamente estudiado mediante Resonancia y Doppler en el paciente de la Figura 1. Nótese preservación de nervio motor.

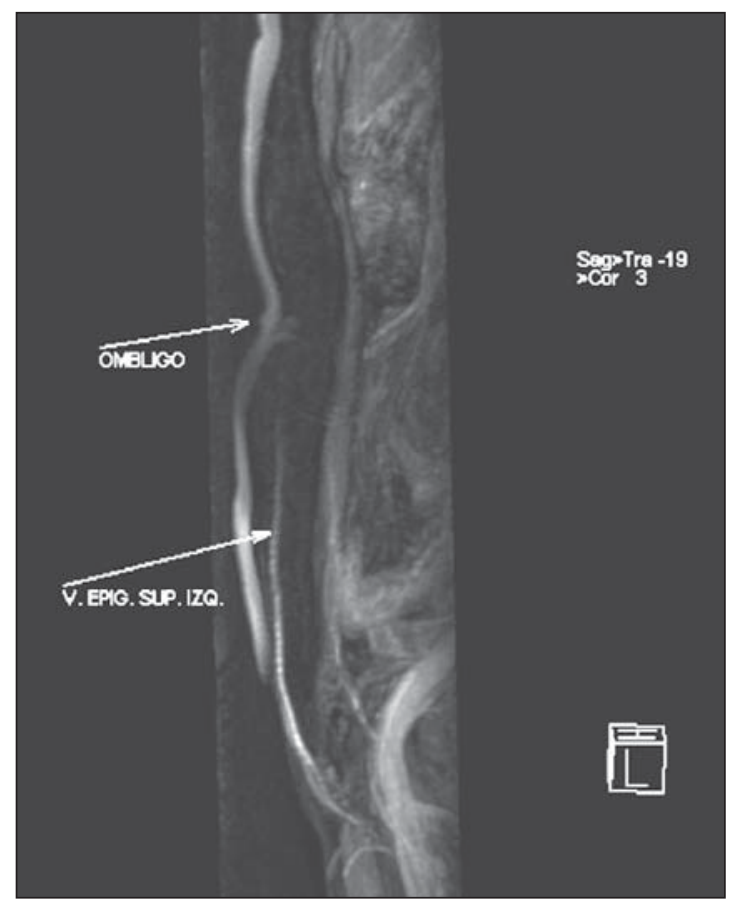

Figura 3. Vaso perforante congruente con estudio de imagen y Doppler.

pleja y prolongando el tiempo intraoperatorio). Esta decisión la basamos en los trabajos de Taylor, que sustentan que hay una relación inversamente proporcional entre el calibre vascular del eje superficial y profundo en la pared abdominal ${ }^{10}$.

Si localizamos una o dos arterias perforantes dominantes (de gran calibre 1,5 a $3 \mathrm{~mm}$ y distribución arborescente por la grasa del colgajo) realizamos un DIEP (Figuras 4, 5, 6). Sólo si hemos evidenciado 
que el trayecto intramuscular es sinuoso y complejo, delegaremos la decisión a un TRAM libre (Figura 7).

Cuando el abdomen que exploramos tienen múltiples perforantes y son finas, o con un trayecto intramuscular complejo realizamos un TRAM MS2, preservando las ramas motoras de los nervios intercostales con el fin de prevenir secuelas importantes en la zona donante.

A todas nuestras pacientes, realizamos un marcaje intraoperatorio con doppler unidireccional, encontrando congruencia en $100 \%$ de los casos con los hallazgos de la angioresonancia, aunque en 2 casos se ha encontrado alguna perforante más de calibre aceptable que no se observó en la prueba de imagen.

Nuestra muestra consta de 25 pacientes, cuyas edades van desde los 32 a 63 años, se realizaron 9

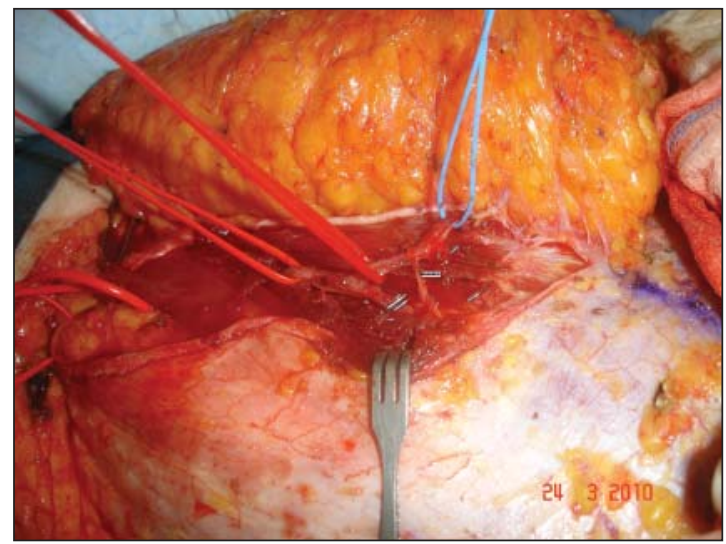

Figura 4. Disección.

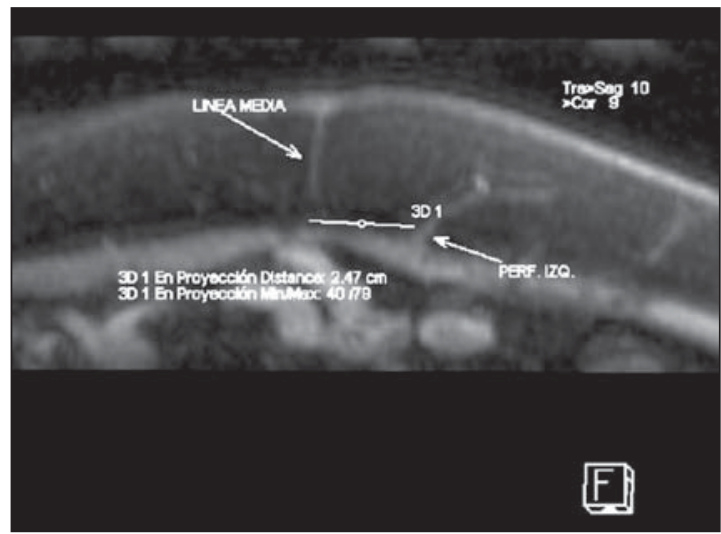

Figura 6. Reconstrucción con algoritmo MAP. Corte sagital en paciente con clara predominancia de sistema epigástrico superficial. Poseía perforante de pequeño calibre decidiéndose finalmente la realización de colgajo SIEA. En la figura se visualiza trayecto de vena epigástrica superficial derecha. reconstrucciones inmediatas y 16 diferidas. Respecto a los colgajos utilizados finalmente, 9 fueron colgajos de perforante de epigástrica inferior profunda, 15 fueron colgajos con pequeña pastilla de recto abdominal y un caso de colgajo de arteria epigástrica inferior superficial.

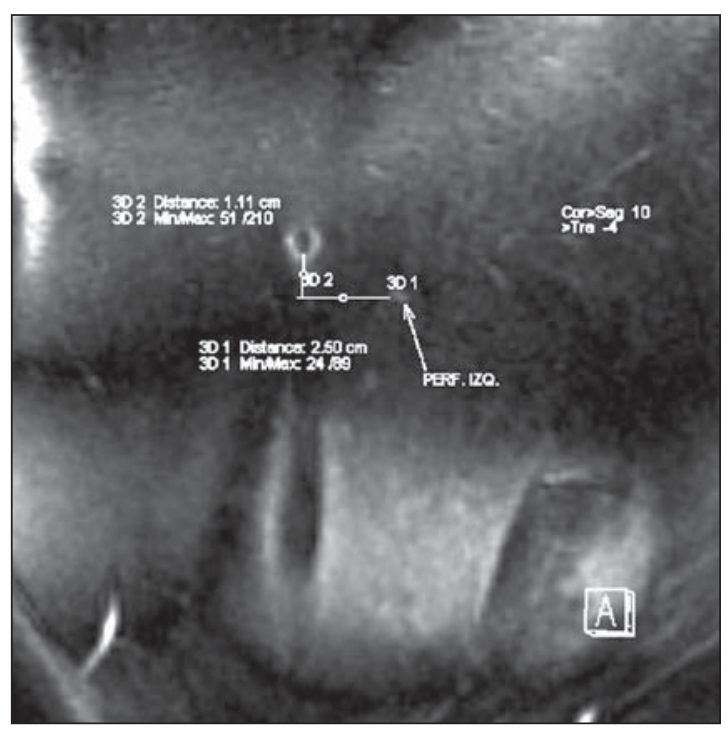

Figura 5. Reconstrucción con algoritmo MAP. Corte transversal donde se visualiza la arteria perforante, su salida desde músculo y arborización por la grasa abdominal.

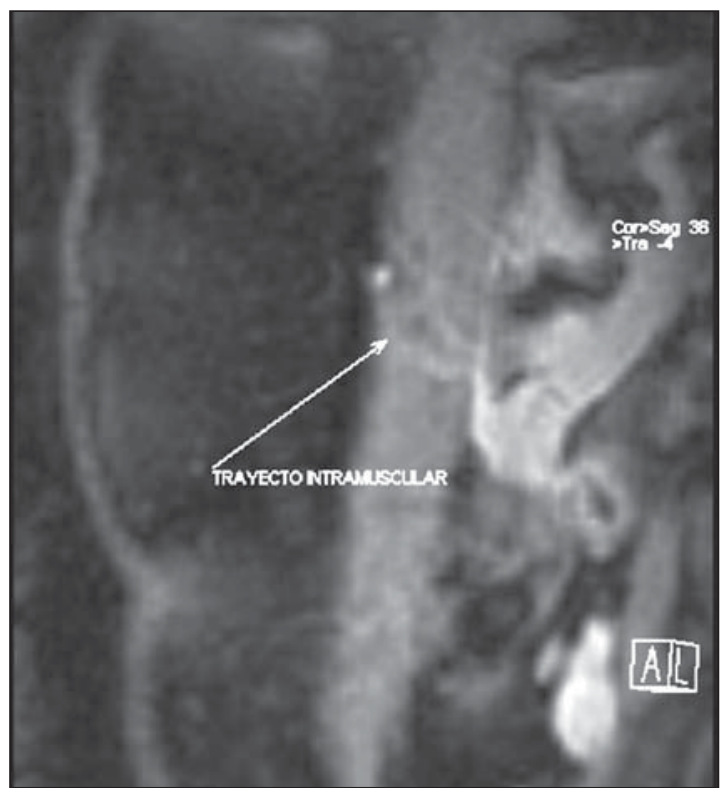

Figura 7. Reconstrucción con algoritmo MAP. Corte sagital donde se observa trayecto intramuscular de la perforante así como su emergencia del recto abdominal y su arborización en la grasa abdominal. 
Trabajamos en dos equipos distintos: uno que prepara los vasos receptores, ya sean los vasos mamarios internos en el $3^{\circ}$ espacio intercostal o los vasos toracodorsales. El otro equipo diseca el colgajo abdominal.

\section{Discusión}

La alta prevalencia de cáncer de mama en el mundo entero así como las secuelas generadas posterior a cirugías con fines oncológicos, lleva consigo la necesidad de realizar reconstrucciones mamarias cada vez con mayor frecuencia. Actualmente el uso de tejido autólogo abdominal, mediante colgajos libres, resulta ser una de las mejores alternativas disponibles.

El estudio mediante ultrasonografía ${ }^{10,11}$ es una forma fácil y no costosa de valorar los vasos perforantes, sin embargo, posee un alto número de falsos positivos y considerables falsos negativos.

Teniendo en cuenta la variabilidad de la vascularización de la pared abdominal, de los vasos perforantes y su diámetro y de su trayecto intramuscular, resulta útil planificar la intervención mediante estudios de imagen previos como la tomografía axial computada y la angioresonancia.

El uso de la angioresonancia permite una valoración previa de las variables ya mencionadas y permite acortar tiempos operatorios así como facilitar la toma de decisiones y la disección de los colgajos. Tiene como beneficio añadido que no irradia a los pacientes, a diferencia de la tomografía axial.

Al analizar las imágenes obtenidas por un radiólogo experto en la técnica, resulta fácil elegir el vaso perforante de mayor calibre para la disección de un colgajo DIEP. Al mismo tiempo la presencia de vasos perforantes de escaso calibre, así como largos trayectos intramusculares, nos orientan a planificar nuestra intervención hacia un colgajo libre TRAM, o la presencia de vasos epigástricos superficiales de características apropiadas definidas por el cirujano y radiólogo nos pueden orientar a realizar la reconstrucción mediante un colgajo libre SIEA.

Respecto al colgajo DIEP, el poder elegir la (s) perforante (s) de mejor calibre y de mejor arborización en la grasa, el cerciorarse de que existe comunicación con la vena epigástrica superficial, y el disecar el pedículo epigástrico inferior profundo hasta que sólo exista una vena, ha hecho que se obtenga un $0 \%$ de problemas de congestión venosa, que habitualmente es una de las complicaciones más frecuente en los colgajos DIEP.

\section{Referencias}

1. Alonso-Burgos A, García-Tutor E, Bastarrika G, Cano D, Martínez-Cuesta A, Piña LJ. Preoperative planning of deep inferior epigastric artery perforator flap reconstruction with multislice-CT angiography: Imaging findings and initial experience. J Plast Reconstr Aesthet Sur 2006; 59: 585-593.

2. Hamdi M, Van Landuyt K, Van Hedent E, Duyck P. Advances in Autogenous Breast Reconstruction. The Role of Preoperative Perforator Mapping. Ann Plast Surg 2007; 58: 18-26.

3. Koshima I, Soeda S. Inferior epigastric artery skin flaps without rectus abdominis muscle. Br J Plast Surg 1989; 42: 645-648.

4. Phillip N, Blondeel KH, Van Landuyt SJ. Monstrey Surgical-technical aspects of the free diep flap for breast reconstruction. Operative Techniques in Plastic and Reconstructive Surgery 1999; 6: 27-37.

5. Lindsey JT. Integrating the DIEP and Muscle-Sparing (MS-2) Free TRAM Techniques Optimizes Surgical Outcomes: Presentation of an Algorithm for Microsurgical Breast Reconstruction Based on Perforator Anatomy. Plast. Reconstr. Surg 2007; 119: 18-27.

6. Taylor G, Daniel R. The anatomy of several free flap donor sites. Plast. Reconstr Surg 1975; 56: 243-253.

7. Grotting J. The free abdominoplasty flap for immediate breast reconstruction. Ann Plast Sur 1991; 27: 351-354.

8. Chevray PM. Breast Reconstruction with Superficial Inferior Epigastric Artery Flaps: A Prospective Comparison with TRAM and DIEP Flaps. Plast Reconstr Surg 2004; 114: 1077-1083.

9. Blondeel N, Vanderstraeten GG, Monstrey SJ, Van Landuyt K, Tonnard P, Lysens R. The donor site morbidity of free DIEP flaps and free TRAM flaps for breast reconstruction. B J Plast Surg 1997; 50: 322330 .

10. Hallock GG. Doppler sonography and colour duplex imaging for planning a perforator flap. Clin Plast Surg 2003; 30: 347-357.

11. Giunta RE, Geisweid A, Feller AM. The value of preoperative Doppler sonography for planning free perforator flaps. Plast Reconstr Surg 2000; 105: 2381-2386.

12. Masia J, Clavero JA, Larranaga JR, Alomar X, Pons G, Serret P. Multidetector-row computed tomography in the planning of abdominal perforator flaps. J Plast Reconstr Aesthet Surg 2006; 59: 594-599.

13. Chernyak V, Rozenblit AM, Greenspun DT, Levine JL, Milikow DL, Chia FA, Erhard HA. Breast Reconstruction with Deep Inferior Epigastric Artery Perforator Flap: 3.0-T Gadolinium enhanced MR Imaging for Preoperative Localization of Abdominal Wall Perforators. Radiology 2009; 250: 417-423. 